\title{
Stage I NSCLC: elucidating the prognostic factors
}

\section{DuyKhanh Pham, David H Harpole}

Non-small cell lung cancer (NSCLC) remains the number one cause of cancer related deaths. ${ }^{1}$ While early stage NSCLC portends the best survival following complete surgical resection, as many as $37 \%$ of patients with stage I disease have recurrence within 5 years of diagnosis. ${ }^{2}$ Several studies have investigated the role of adjuvant chemotherapy for early stage disease. ${ }^{3-6}$ However, the benefit of adjuvant therapy was mainly in patients with stage II and IIIA disease. Significant progress in improving the survival of patients with stage I NSCLC is lacking.

In this issue, Hung and colleagues ${ }^{7}$ address two very important issues with regard to the management of early stage NSCLC (see page 192): (1) the prognostic predictors of survival in patients with recurrence and (2) the optimal treatment for recurrent disease. In this retrospective study of 970 patients with resected stage I NSCLC, the clinicopathological characteristics of 123 patients with recurrence were analysed for prognostic indicators of postrecurrence survival. Seventy-four patients had local recurrence only, and 1 and 2 year survival following recurrence was $48.7 \%$ and $17.6 \%$, respectively. Of several clinical factors analysed, tumour size $(p=0.0033)$ and initial treatment of recurrence $(p<0.001)$ were significant predictors of post-recurrence survival in univariate analysis. In multivariate analysis, initial treatment of recurrence remained a significant prognostic indicator of survival ( $p=0.001$ ). Patients receiving surgery as initial treatment had a better survival than those treated with chemotherapy and/or radiotherapy. Moreover, patients receiving chemotherapy and/or radiotherapy did better than those who received no treatment.

While this study suffers from the usual pitfalls of small, retrospective studies, Hung and colleagues ${ }^{7}$ should be commended for investigating not only the natural history and survival of patients with stage I NSCLC but also the prognostic

Division of Thoracic Surgery, Duke University Health System, Durham, North Carolina, USA

Correspondence to: Dr David H Harpole jr, Duke University Medical Center, Box 3627, Durham, NC 27710, USA; harpo002@mc.duke.edu predictors of survival following recurrence. The authors bring to light a very important population of patients with potentially curable disease to remind us that our success rate with stage I NSCLC can be improved.

To a similar end, several groups have performed clinical and histological studies on various patients with stage I NSCLC to identify those who are at higher risk of recurrence and who, possibly, would benefit from aggressive adjuvant therapy following resection. In a case matched retrospective study, Brock and colleagues ${ }^{8}$ distinctly identified a correlation between DNA methylation of the promoters of the cyclin dependent kinase inhibitor 2A gene 16, H-cadherin gene CDH13, Ras association gene RASSF1A and adenomatous polyposis coli gene with early tumour recurrence. Immunohistochemistry staining of vascular endothelial growth factor (VEGF) has been found to be a prognostic factor for time to relapse and survival in patients with early stage disease. ${ }^{9} 10$ Increased carcinoembryonic antigen levels and Ki67 labelling index is associated with decreased survival and early recurrence. ${ }^{11}{ }^{12}$ Additionally, the presence of tumour regulatory $\mathrm{T}$ cells and infiltrating-infiltrating $\mathrm{T}$ cells in the primary lesion has been significantly correlated with a higher risk of recurrence. ${ }^{13}$

An equally fervent group of researchers focusing on genomics have identified gene expression profiles and metagene signatures that predict recurrence in patients with early stage NSCLC. ${ }^{14-18}$ Jiang and colleagues $^{14}$ identified gene clusters via comparative genomic hybridisation and cDNA microarray analysis that may be involved in the initiation and progression of NSCLC. Potti and colleagues ${ }^{15}$ developed a lung metagene model that successfully identified subgroups of patients who were at increased risk of recurrence with an accuracy as high as $79 \%$. Larsen and colleagues ${ }^{16}$ identified a 54 gene expression signature capable of predicting poor outcome groups of patients despite favourable clinical factors.

Collectively, these studies highlight the challenge of treating patients with stage I NSCLC. While these patients are seemingly low risk, the population is in fact quite heterogeneous with a subset of patients at higher risk of recurrence and decreased survival. The crux of treating these patients is accurate identification of the high risk patients and aggressive treatment with individualised adjuvant therapy. ${ }^{17}{ }^{18}$ As Hung and colleagues ${ }^{7}$ illustrate in their concise study, risk stratification and aggressive management (with possible surgical management when appropriate) of patients with recurrent disease offers the best chance of improved outcomes. In managing patients in this manner, we anticipate significant progress in the survival of patients with stage I NSCLC.

\section{Competing interests: None.}

Thorax 2009;64:185-186. doi:10.1136/thx.2008.105965

\section{REFERENCES}

1. Jemal A, Siegel R, Ward E, et al. Cancer statistics, 2008. CA Cancer J Clin 2008;58:71-96.

2. Harpole DH Jr, Herndon JE, Young WG, et al. Stage I non-small cell lung cancer. Cancer 1995:76:787-96.

3. IALT Collaborative Group. Cisplatin-based adjuvant chemotherapy in patients with completely resected non-small-cell lung cancer. $N$ Engl J Med 2004;350:351-60.

4. Winton T, Livington R, Johnson D, et al. Vinorelbine plus cisplatin vs. observation in resetced non-smallcell lung cancer. N Engl J Med 2005;352:2589-97.

5. Strauss GM, Herndon JE, Maddaus MA, et al. Adjuvant paclitaxel plus carboplatin compared with observation in stage IB non-small-cell lung cancer: CALGB 9633 with the Cancer and Leukemia Group B, Radiation Therapy Oncology Group, and North Central Cancer Treatment Group Study Groups. J Clin Oncol 2008;26:5043-51.

6. Douillard JY, Rossell R, De Lena M, et al. Adjuvant vinorelbine plus cisplatin versus observation in patients with completely resected stage IB-IIIA nonsmall-cell lung cancer (Adjuvant Navelbine International Trialist Association [ANITA]): a randomised controlled trial. Lancet Oncol 2006:7:719-27.

7. Hung J-J, Hsu W-H, Hsieh C-C, et al. Post-recurrence survival in completely resected stage I non-small cell lung cancer with local recurrence. Thorax 2009:64:192-6.

8. Brock MV, Hooker CM, Ota-Machida E, et al. DNA methylation markers and early recurrence in stage I lung cancer. N Engl J Med 2008 13;358:1118-28.

9. Ohta T, Tanaka Y, Watannabe G, et al. Predicting recurrence following curative surgery in stage I nonsmall cell lung cancer patients using an angiogenesisassociated factor. J Exp Clin Cancer Res 2007;26:301-5

10. Yilmaz A, Ernam D, Unsal E, et al. Vascular endothelial growth factor immunostaining correlates with postoperative relapse and survival in non-small cell lung cancer. Arch Med Res 2007;38:764-8.

11. Matsuoka K, Sumitomo S, Nakashima N, et al. Prognostic value of carcinoembryonic antigen and CYFRA21-1 in patients with pathological stage I nonsmall cell lung cancer. Eur J Cardiothorac Surg 2007;32:435-9

12. Inoue M, Takakuwa $\mathrm{T}$, Minami $\mathrm{M}$, et al. Clinicopathologic factors influencing postoperative prognosis in patients with small-sized adenocarcinoma of the lung. $J$ Thorac Cardiovasc Surg 2008;135:830-6.

13. Petersen RP, Campa MJ, Sperlazza J, et al. Tumor infiltrating Foxp3+ regulatory T-cells are associated 
with recurrence in pathologic stage I NSCLC patients. Cancer 2006;107:2866-72.

14. Jiang F, Yin Z, Caraway NP, et al. Genomic profiles in stage I primary non small cell lung cancer using comparative genomic hybridization analysis of cDNA microarrays. Neoplasia 2004;6:623-35.
15. Potti A, Mukherjee S, Petersen R, et al. A genomic strategy to refine prognosis in early-stage non-smallcell lung cancer. N Engl J Med 2006;355:570-80.

16. Larsen JE, Pavey SJ, Passmore LH, et al. Gene expression signature predicts recurrence in lung adenocarcinoma. Clin Cancer Res 2007;13:2956-64.
17. Harpole DH. Prognostic modeling in early stage lung cancer: an evolving process from histopathology to genomics. Thor Surg Clin 2007:17:167-73, vii.

18. Anguiano A, Nevins JR, Potti A. Toward the individualization of lung cancer therapy. Cancer 2008;113(7 Suppl):1760-7.

\section{Azithromycin therapy for neutrophilic airways disease: myth or magic?}

\section{Saad F Idris, ${ }^{1}$ Edwin R Chilvers, ${ }^{1}$ Charles Haworth, ${ }^{2}$ Damian McKeon, ${ }^{1,2}$ Alison M Condliffe ${ }^{1}$}

The anti-inflammatory potential of macrolides was first appreciated with the successful use of erythromycin in the treatment of diffuse panbronchiolitis, a disease principally affecting the Japanese, and characterised by a persistent neutrophilic inflammatory infiltrate of the bronchi. While there are only limited data on the efficacy of other macrolides in treating this condition, ${ }^{1}$ the observation has generated considerable interest in examining the role of macrolides in other respiratory diseases where chronic airways inflammation is a prominent feature.

Azithromycin (AZM) is a 15-membered macrolactam ring macrolide which, in randomised controlled studies, has demonstrated a beneficial role in the treatment of cystic fibrosis $(\mathrm{CF})^{2-5}$ despite its lack of direct bactericidal or bacteriostatic activity against Pseudomonas aeruginosa. More recent (albeit smaller) studies have suggested a role for AZM in the treatment of bronchiolitis obliterans syndrome (BOS) ${ }^{6}$ and asthma, ${ }^{7}$ with a common finding in both being its ability to reduce airway neutrophilia. Such observations have focused attention on understanding how AZM and other macrolides may modulate host-pathogen interactions in chronic lung infection and their role as an immunomodulatory agent in both respiratory and nonrespiratory settings. Clinical studies have

\footnotetext{
${ }^{1}$ Respiratory Medicine Division, Department of Medicine, University of Cambridge School of Clinical Medicine, Cambridge, UK; ${ }^{2}$ Adult Cystic Fibrosis Unit, Papworth Hospital, Cambridge, UK

Correspondence to: Dr A M Condliffe, Respiratory Medicine Division, Department of Medicine, University of Cambridge School of Clinical Medicine, Box 157 Addenbrooke's Hospital, Cambridge University Hospitals NHS Foundation Trust, Hills Road, Cambridge CB2 200 UK; amc46@cam.ac.uk
}

usually been designed to study an individual macrolide agent, but it is likely that the findings in such trials represent a "class effect". Although some in vitro experiments have suggested subtle differences between individual class members, the in vivo significance of these observations is uncertain.

\section{MODULATION OF HOST-PATHOGEN INTERACTIONS}

The direct antimicrobial activity of macrolides against Gram-positive bacteria results from inhibition of bacterial protein synthesis. Although AZM has little direct activity against Gram-negative organisms, it has been shown to modulate bacterial virulence factors and thus affect the outcome of chronic infections with organisms such as $P$ aeruginosa. Quorum sensing is a sophisticated mechanism whereby pathogen-derived molecules act as auto-inducers and trigger a variety of biological functions such as biofilm formation and production of virulence factors. AZM reduces the transcription of lasI and rhlI, two vital components of the quorum sensing system, resulting in reduced generation of the auto-inducer molecule HSL. ${ }^{8}$ Furthermore, in vitro studies have shown that AZM-exposed $P$ aeruginosa displays impaired mobility and oxidative stress responses, ${ }^{9}$ perhaps contributing to the reduced biofilm formation observed in AZM-treated cultures. ${ }^{10}$ More recently, sub-MIC concentrations of AZM have also been shown to decrease formation of Haemophilus influenzae biofilms. ${ }^{1}$

In CFTR-null mice infected with $P$ aeruginosa, AZM suppressed the production of virulence factors, improved the clearance of $P$ aeruginosa biofilms and reduced the severity of lung pathology. ${ }^{12}$ In a separate study conducted in wild-type mice, AZM failed to affect the pulmonary clearance of $P$ aeruginosa embedded in agar beads but dramatically reduced the cellular infiltrate in the lung, with a substantial reduction in neutrophil numbers. ${ }^{13}$ The disparity between these studies probably reflects methodological differences, not least the higher dose of AZM used in the former study (500 mg/kg vs $20 \mathrm{mg} / \mathrm{kg}$ ) and the use of suspension rather than agarembedded organisms which are more likely to promote biofilm production. Taken together, these studies indicate that AZM is able to antagonise bacterial virulence in the absence of a direct antibacterial effect; however, its beneficial effects in lung inflammation may not be restricted to modulating host-pathogen interactions. This view is supported by findings in mice homozygous for the $\Delta \mathrm{F} 508$ mutation, where AZM reduced spontaneous and lipopolysaccharide (LPS)-induced inflammatory changes, suggesting that AZM may have direct effects on the immune system. ${ }^{14}$

\section{MODULATION OF CYTOKINE RESPONSES}

Several studies have demonstrated the ability of macrolide antibiotics, including AZM, to modulate cytokine responses both in vitro and in vivo. AZM significantly reduced nuclear factor-kappa B (NF- $\mathrm{KB}$ ) expression, tumour necrosis factor $\alpha(T N F \alpha)$ mRNA levels and TNF $\alpha$ secretion in a CF-derived airway epithelial cell line. ${ }^{15}$ In mice treated with intraperitoneal LPS, AZM attenuated the increase in plasma TNF $\alpha$ and increased the survival in animals challenged with intravenous LPS. ${ }^{16}$ Together, these studies show that AZM can ameliorate the proinflammatory response to bacterial antigens. This conclusion is supported by work examining the role of AZM in the treatment of bronchopulmonary dysplasia (BPD), which has demonstrated suppression of $\mathrm{TNF} \alpha$-stimulated $\mathrm{NF}-\kappa \mathrm{B}$ activation in tracheal aspirate cells from preterm infants with the reduction of interleukin (IL)- 6 and IL-8 production to control levels. ${ }^{17}$ Similarly, in an animal model of BPD, AZM caused decreased IL-6 production, less emphysematous change 